ANNALES

POLONICI MATHEMATICI

LXXVIII.3 (2002)

\title{
On the existence for the Dirichlet problem for the compressible linearized Navier-Stokes system in the $L_{p}$-framework
}

\author{
by Piotr Boguseaw Mucha and \\ WOJCIECH ZAJĄCZKOWSKI (Warszawa)
}

\begin{abstract}
The existence of solutions to the Dirichlet problem for the compressible linearized Navier-Stokes system is proved in a class such that the velocity vector belongs to $W_{r}^{2,1}$ with $r>3$. The proof is done in two steps. First the existence for local problems with constant coefficients is proved by applying the Fourier transform. Next by applying the regularizer technique the existence in a bounded domain is shown.
\end{abstract}

1. Introduction. In a bounded domain $\Omega$ in $\mathbb{R}^{3}$ with boundary $S$ we consider the initial-boundary value problem for the compressible linearized Navier-Stokes system:

$$
\begin{aligned}
& C u_{t}-\mu \Delta u-\nu \nabla \operatorname{div} u+A \nabla \eta=F, \\
& \eta_{t}+B \operatorname{div} u=H, \\
& \left.u\right|_{S_{T}}=G, \\
& \left.u\right|_{t=0}=u_{0},\left.\quad \eta\right|_{t=0}=\eta_{0},
\end{aligned}
$$

where $u(x, t)=\left(u_{1}(x, t), u_{2}(x, t), u_{3}(x, t)\right)$ is the velocity vector, $\eta(x, t)$ the density, $\mu, \nu$ constant positive viscosity coefficients and $A(x, t), B(x, t)$, $C(x, t)$ positive functions from $C^{\alpha}(\Omega \times[0, T])$.

We prove the existence of solutions for system (1.1). The main result of this paper is the following

Theorem 1. Let $r>3, S \in W_{r}^{2-1 / r}, F \in L_{r}\left(\Omega_{T}\right), H \in W_{r}^{1,0}\left(\Omega_{T}\right)$, $G \in W_{r}^{2-1 / r, 1-1 /(2 r)}\left(S_{T}\right), u_{0} \in W_{r}^{2-2 / r}(\Omega), \eta_{0} \in W_{r}^{1}(\Omega), A, B, C \in C^{\alpha}(\bar{\Omega} \times$

2000 Mathematics Subject Classification: 35Q30, 76Q10.

Key words and phrases: compressible Navier-Stokes system, Dirichlet problem, $L_{p^{-}}$ framework.

Supported by Polish KBN Grant 2 P03A 03816. 
$[0, T])$ with $\alpha>0$ and

$$
0<C_{*} \leq A(x, t), B(x, t), C(x, t) \leq C^{*}<\infty .
$$

Then there exists a unique solution of problem (1.1) such that

$$
u \in W_{r}^{2,1}\left(\Omega_{T}\right), \quad \eta \in W_{r}^{1,0}\left(\Omega_{T}\right), \quad \eta_{t} \in W_{r}^{1,0}\left(\Omega_{T}\right)
$$

and the following estimate holds:

$$
\begin{aligned}
& \|u\|_{W_{r}^{2,1}\left(\Omega_{T}\right)}+\|\eta\|_{W_{r}^{1,0}\left(\Omega_{T}\right)}+\left\|\eta_{t}\right\|_{W_{r}^{1,0}\left(\Omega_{T}\right)} \\
& \leq C(T)\left[\|f\|_{L_{r}\left(\Omega_{T}\right)}+\|H\|_{W_{r}^{1,0}\left(\Omega_{T}\right)}+\|G\|_{W_{r}^{2-1 / r, 1-1 /(2 r)}\left(S_{T}\right)}\right. \\
& \left.\quad+\left\|u_{0}\right\|_{W_{r}^{2-2 / r}(\Omega)}+\left\|\eta_{0}\right\|_{W_{r}^{1,0}(\Omega)}\right]
\end{aligned}
$$

where $\Omega_{T}=\Omega \times(0, T), S_{T}=S \times(0, T)$ and $C(T)$ is an increasing function of $T$.

The proof of Theorem 1 is divided into two steps. In the first step the existence for the corresponding local problems with constant coefficients is proved by applying the Fourier transform. In the second step the regularizer technique is used to prove the existence in a bounded domain and with nonvanishing initial data.

In the first step after localizing system (1.1) and freezing its coefficients we apply the Fourier transform to obtain a system of ordinary differential equations. Solving them explicitly we estimate them directly applying the Marcinkiewicz theorem $[2,3]$. Hence we omit the difficulties with constructing and estimating the corresponding potentials.

Our estimates are new, quite simple and original. We think that it is an interesting direction.

2. Notation. In our considerations we will need the anisotropic Sobolev spaces $W_{r}^{m, n}\left(Q_{T}\right)$ where $m, n \in \mathbb{R}_{+} \cup\{0\}, r \geq 1$ and $Q_{T}=Q \times(0, T)$ with the norm

$$
\begin{aligned}
& \|u\|_{W_{r}^{m, n}\left(Q_{T}\right)}^{r}=\int_{0}^{T} \int_{Q}|u(x, t)|^{r} d x d t+\sum_{0 \leq\left|m^{\prime}\right| \leq[|m|]} \int_{0}^{T} \int_{Q}\left|D_{x}^{m^{\prime}} u(x, t)\right|^{r} d x d t \\
& +\sum_{\left|m^{\prime}\right|=[|m|]} \int_{0}^{T} d t \int_{Q} \int_{Q} \frac{\left|D_{x}^{m^{\prime}} u(x, t)-D_{x}^{m^{\prime}} u\left(x^{\prime}, t\right)\right|^{r}}{\left|x-x^{\prime}\right|^{s+r(|m|-[|m|])}} d x d x^{\prime} \\
& +\sum_{0 \leq\left|n^{\prime}\right| \leq[|n|]} \int_{0}^{T} \int_{Q}\left|D_{t}^{n^{\prime}} u(x, t)\right|^{r} d x d t \\
& +\int_{Q} d x \int_{0}^{T} \int_{0}^{T} \frac{\left|D_{t}^{[n]} u(x, t)-D_{t^{\prime}}^{[n]}\left(x, t^{\prime}\right)\right|^{r}}{\left|t-t^{\prime}\right|^{1+r(n-[n])}} d t d t^{\prime}
\end{aligned}
$$


where $s=\operatorname{dim} Q,[\alpha]$ is the integral part of $\alpha$, and $D_{x}^{l}=\partial_{x_{1}}^{l_{1}} \ldots \partial_{x_{s}}^{l_{s}}$ where $l=\left(l_{1}, \ldots, l_{s}\right)$ is a multiindex. If $Q$ is a manifold the above norms are defined by using a partition of unity.

In the case when $Q_{T}=\mathbb{R}^{s} \times \mathbb{R}$ we can apply the Fourier transform and

$$
\begin{aligned}
& \|u\|_{H_{r}^{m, n}\left(\mathbb{R}^{s+1}\right)}=\|u\|_{L_{r}\left(\mathbb{R}^{s+1}\right)} \\
& \quad+\left\|\mathcal{F}_{t, x}^{-1}\left[|\xi|^{m} \widehat{u}\left(\xi, \xi_{0}\right)\right]\right\|_{L_{r}\left(\mathbb{R}^{s+1}\right)}+\left\|\mathcal{F}_{t, x}^{-1}\left[\left|\xi_{0}\right|^{n} \widehat{u}\left(\xi, \xi_{0}\right)\right]\right\|_{L_{r}\left(\mathbb{R}^{s+1}\right)}
\end{aligned}
$$

where $\widehat{u}\left(\xi, \xi_{0}\right)$ is the Fourier transform of $u(x, t)$,

$$
\widehat{u}\left(\xi, \xi_{0}\right)=\int e^{-i \xi_{0} t} \int e^{-i \xi \cdot x} u(x, t) d x d t \equiv \mathcal{F}_{t, x}[u]\left(\xi, \xi_{0}\right)
$$

and $\mathcal{F}^{-1}$ the inverse transform

$$
\mathcal{F}_{t, x}^{-1}[\widehat{u}](x, t)=(2 \pi)^{-2(s+1)} \int e^{i \xi_{0} t} \int e^{i \xi \cdot x} \widehat{u}\left(\xi, \xi_{0}\right) d \xi d \xi_{0}
$$

where $\xi=\left(\xi_{1}, \ldots, \xi_{s}\right)$ and $\xi \cdot x=\xi_{1} x_{1}+\ldots+\xi_{s} x_{s}$.

We also define the space $V\left(Q_{T}\right)$ as the closure

$$
V\left(Q_{T}\right)=\bar{C}^{\infty}\left(Q_{T}\right)\|\cdot\|_{V\left(Q_{T}\right)},
$$

where

$$
\|u\|_{V\left(Q_{T}\right)}=\|u\|_{W_{r}^{1,0}\left(Q_{T}\right)}+\left\|u_{t}\right\|_{W_{r}^{1,0}\left(Q_{T}\right)} .
$$

In the proof we will use the following results.

Theorem 2.1 (Marcinkiewicz theorem; see [3]). Suppose that the function $\Phi: \mathbb{R}^{m} \rightarrow \mathbb{C}$ is smooth enough and there exists $M>0$ such that for every $x \in \mathbb{R}^{m}$ we have

$$
\left|x_{j_{1}} \ldots x_{j_{k}}\right|\left|\frac{\partial^{k} \Phi}{\partial x_{j_{1}} \ldots \partial x_{j_{k}}}\right| \leq M, \quad 0 \leq k \leq m, 1 \leq j_{1}<\ldots<j_{k} \leq m .
$$

Then the operator

$$
\operatorname{Pg}(x)=(2 \pi)^{-m} \int_{\mathbb{R}^{m}} d y e^{i x y} \Phi(y) \int_{\mathbb{R}^{m}} e^{-i y z} g(z) d z
$$

is bounded in $L_{p}\left(\mathbb{R}^{m}\right)$ and

$$
\|P g\|_{L_{p}\left(\mathbb{R}^{m}\right)} \leq A_{p, m} M\|g\|_{L_{p}\left(\mathbb{R}^{m}\right)} .
$$

Proposition 2.2 (see [5]). If $r>2$ and $m, n>0$ then $H_{r}^{m, n}\left(\mathbb{R}^{s} \times \mathbb{R}\right) \subset$ $W_{r}^{m, n}\left(\mathbb{R}^{s} \times \mathbb{R}\right)$, and

$$
\|u\|_{W_{r}^{m, n}\left(\mathbb{R}^{s} \times \mathbb{R}\right)} \leq c\|u\|_{H_{r}^{m, n}\left(\mathbb{R}^{s} \times \mathbb{R}\right)} .
$$

Moreover if $m, n \in \mathbb{N}$ then $H_{r}^{m, n}=W_{r}^{m, n}$. 
Proposition 2.3 (see [1]). Let $u \in W_{r}^{m, n}\left(\Omega_{T}\right), m, n \in \mathbb{R}_{+}$and $q \geq r$. Suppose that

$$
\kappa=\sum_{i=1}^{3}\left(\alpha_{i}+\frac{1}{r}-\frac{1}{q}\right) \frac{1}{m}+\left(\beta+\frac{1}{r}-\frac{1}{q}\right) \frac{1}{n}<1 .
$$

Then

$$
\left\|D_{t}^{\beta} D_{x}^{\alpha} u\right\|_{L_{q}\left(\Omega_{T}\right)} \leq \varepsilon^{1-\kappa}\|u\|_{W_{r}^{m, n}\left(\Omega_{T}\right)}+c \varepsilon^{-\kappa}\|u\|_{L_{r}\left(\Omega_{T}\right)}
$$

for all $\varepsilon \in(0,1)$.

Proposition 2.4. Let $r \geq 2, u \in W_{r}^{2,1}\left(\Omega_{T}\right)$ and $\left.u\right|_{t=0}=0$. Then

$$
\|u\|_{W_{r}^{1,1 / 2}\left(\Omega_{T}\right)} \leq c\left(T^{1 / r}+T^{1 / 2}\right)\|u\|_{W_{r}^{2,1}\left(\Omega_{T}\right)} .
$$

Proof. First we note that

$$
\begin{aligned}
& \iint_{\Omega}^{T} \int_{0}^{T} \frac{\left|u(x, t)-u\left(x, t^{\prime}\right)\right|^{r}}{\left|t-t^{\prime}\right|^{1+r / 2}} d t^{\prime} d t d x \\
& \quad \leq T^{r / 2} \int_{\Omega}^{T} \int_{0}^{T} \int_{0}^{T} \frac{\left|u(x, t)-u\left(x, t^{\prime}\right)\right|^{r}}{\left|t-t^{\prime}\right|^{1+r}} d t^{\prime} d t d x \leq T^{r / 2}\|u\|_{W_{r}^{0,1}\left(\Omega_{T}\right)}^{r} .
\end{aligned}
$$

Next we have

$$
\begin{aligned}
\|u\|_{L_{r}\left(\Omega_{T}\right)} & \leq\|u\|_{W_{r}^{1,0}\left(\Omega_{T}\right)} \\
& \leq T^{1 / r}\|u\|_{L_{\infty}\left(0, T ; L_{r}(\Omega)\right)} \leq T^{1 / r}\|u\|_{W_{r}^{2,1}\left(\Omega_{T}\right)},
\end{aligned}
$$

where in the last inequality we use $\left.u\right|_{t=0} \equiv 0$. From (2.4) and (2.5) we get the assertion.

During our considerations we will use well known results such as the imbedding theorems for Sobolev spaces. All constants are denoted by $c$.

3. Problem in the half space. We consider the linear system

$$
\begin{aligned}
& u_{t}-\mu \Delta u-\nu \nabla \operatorname{div} u+a \nabla \eta=f, \\
& \eta_{t}+b \operatorname{div} u=h, \\
& \left.u\right|_{x_{3}=0}=g, \\
& \left.u\right|_{t=0}=0,\left.\quad \eta\right|_{t=0}=0, \\
& u, \eta \rightarrow 0 \quad \text { as } x_{3} \rightarrow \infty
\end{aligned}
$$

in the half space $x_{3} \geq 0$. To solve (3.1) we need some results for the heat equation.

To simplify the considerations we assume that all the given data are smooth. The desired result will follow by a limit passage. 
We examine the following problem in the half space:

$$
\begin{aligned}
& w_{t}-(\mu+\nu) \Delta w=\operatorname{div} f, \\
& \left.w\right|_{x_{3}=0}=w_{0}, \\
& \left.w\right|_{t=0}=0, \\
& w \rightarrow 0 \quad \text { as } x_{3} \rightarrow \infty .
\end{aligned}
$$

Lemma 3.1. Let $f \in L_{r}\left(\mathbb{D}^{4}\right)$ and $w_{0} \in W_{r}^{1-1 / r, 1 / 2-1 /(2 r)}\left(\mathbb{R}^{3}\right)$. Then there exists a unique solution of problem (3.2) such that $w \in W_{r}^{1,0}\left(\mathbb{D}_{T}^{4}\right)$ and the following estimate holds:

$$
\|\nabla w\|_{L_{r}\left(\mathbb{D}_{T}^{4}\right)} \leq c(T)\left(\|f\|_{L_{r}\left(\mathbb{D}_{T}^{4}\right)}+\left\|w_{0}\right\|_{W_{r}^{1-1 / r, 1 / 2-1 /(2 r)}\left(\mathbb{R}_{T}^{3}\right)}\right),
$$

where $\mathbb{D}^{4}=\mathbb{R}_{x^{\prime}} \times[0, \infty)_{x_{3}} \times[0, T]_{t}$.

Proof. First we consider (3.2) in the homogeneous case:

$$
\begin{aligned}
& w_{t}-(\mu+\nu) \Delta w=0, \\
& \left.w\right|_{x_{3}=0}=\phi, \\
& \left.w\right|_{t=0}=0, \\
& w \rightarrow 0 \quad \text { as } x_{3} \rightarrow \infty,
\end{aligned}
$$

where $\phi \in W_{r}^{1-1 / r, 1 / 2-1 /(2 r)}\left(\mathbb{R}^{3}\right)$.

Applying the Fourier transform to $(3.4)_{1}$ yields

$$
r^{\prime 2} w-w_{, 33}=0,
$$

where $r^{\prime}=\sqrt{i \xi_{0} /(\mu+\nu)+\left|\xi^{\prime}\right|^{2}}$, and using (3.4) 4 we get a solution of (3.4):

$$
w=\mathcal{F}_{t, x^{\prime}}^{-1}\left[\phi e^{-r^{\prime} x_{3}}\right] \text {. }
$$

We have to show that $w \in W_{r}^{1,0}$. In [4, Lemma 3.2] we have shown that

$$
\left\|\partial_{x^{\prime}} \mathcal{F}_{t, x^{\prime}}^{-1}\left[\phi e^{-r^{\prime} x_{3}}\right]\right\|_{L_{r}\left(\mathbb{D}^{4}\right)} \leq c\|\phi\|_{W_{r}^{1-1 / r, 1 / 2-1 /(2 r)}\left(\mathbb{R}^{3}\right)} .
$$

Here we only estimate $\partial_{3} w$, which was not explicitly given in [4]. We have

$$
\partial_{3} w=-\mathcal{F}_{t, x^{\prime}}^{-1}\left[\phi r^{\prime} e^{-r^{\prime} x_{3}}\right]=-\mathcal{F}_{t, x^{\prime}}^{-1}\left(\frac{i \xi_{0}}{\mu+\nu}+\left|\xi^{\prime}\right|^{2}\right) \frac{e^{-r^{\prime} x_{3}}}{r^{\prime}} \phi .
$$

Estimates for $\left|\xi^{\prime}\right|^{2}\left(e^{-r^{\prime} x_{3}} / r\right) \phi$ can be found in [4] or deduced from (3.5). Here we examine only

$$
I=\mathcal{F}_{t, x^{\prime}}^{-1}\left[\xi_{0} \frac{e^{-r^{\prime} x_{3}}}{r^{\prime}} \phi\right]=c \partial_{t} \mathcal{F}_{t, x^{\prime}}^{-1}\left[\frac{e^{-r^{\prime} x_{3}}}{r^{\prime}} \phi\right]=c \partial_{t} \Gamma * \phi=c \Gamma_{t} * \phi
$$

where

$$
\Gamma(t, x)=\frac{1}{(4 \pi(\mu+\nu) t)^{3 / 2}} e^{-|x|^{2} /(4(\mu+\nu) t)} .
$$


We know that $\int d t \Gamma_{t}=0$, so we have

$$
I(t, x)=\int_{0}^{\infty} d t \int d y^{\prime} \Gamma_{t}\left(t^{\prime}, y^{\prime}, x_{3}\right)\left[\phi\left(t-t^{\prime}, x^{\prime}-y^{\prime}\right)-\phi\left(t, x^{\prime}-y^{\prime}\right)\right],
$$

where

$$
\begin{aligned}
\Gamma_{t}= & -\frac{3}{2} \cdot \frac{1}{(4 \pi(\mu+\nu))^{3 / 2}} \cdot \frac{1}{t^{5 / 2}} e^{-|x|^{2} /(4(\mu+\nu) t)} \\
& +\frac{1}{(4 \pi(\mu+\nu))^{3 / 2}} \cdot \frac{|x|^{2}}{4(\mu+\nu) t^{7 / 2}} e^{-|x|^{2} /(4(\mu+\nu) t)}=K_{1}+K_{2} .
\end{aligned}
$$

Then we have

$$
\|I\|_{L_{r}\left(\mathbb{R}^{3}\right)} \leq \int d t \int d y^{\prime}\left(\left|K_{1}\right|+\left|K_{2}\right|\right) N(t)=I_{1}+I_{2},
$$

where

$$
N(t)=\left\|\phi\left(x^{\prime}-y^{\prime}, t^{\prime}-t\right)-\phi\left(x^{\prime}-y^{\prime}, t^{\prime}\right)\right\|_{L_{r}\left(\mathbb{R}_{y^{\prime}}^{2} \times \mathbb{R}_{t^{\prime}}\right)} .
$$

Now,

$$
\begin{aligned}
I_{1} \leq & c\left(\int d t \int d x^{\prime} \frac{1}{t^{(5 / 2-m) r}} e^{-|x|^{2} /(4(\mu+\nu) t)} N^{r}(t)\right)^{1 / r} \\
& \times\left(\int d t \int d x^{\prime} \frac{1}{t^{m r^{*}}} e^{-|x|^{2} /(4(\mu+\nu) t)}\right)^{1 / r^{*}}
\end{aligned}
$$

where $r^{*}=r /(r-1)$. We consider the last integral

$$
J_{1}=\int d t \int d x^{\prime} \frac{1}{t^{m r^{*}}} e^{-|x|^{2} /(4(\mu+\nu) t)}=\int d t t^{1-m r^{*}} e^{-x_{3}^{2} /(4(\mu+\nu) t)} .
$$

Taking $w=x_{3}^{2} /(4(\mu+\nu) t)$ we obtain

$$
J_{1}=c \int d w \frac{x_{3}^{2}}{w^{2}}\left(\frac{x_{3}^{2}}{w}\right)^{1-m r^{*}} e^{-w}=c x_{3}^{2+2-2 m r^{*}} \int d w w^{-2-1+m r^{*}} e^{-w} .
$$

For finiteness of $J_{1}$ we need $-3+m r^{*}>-1$ or

$$
m>2-\frac{2}{r}
$$

Thus we get

$$
J_{1}=c x_{3}^{4-2 m r^{*}}
$$

We have

$$
\left(4-2 m r^{*}\right) \frac{r}{r^{*}}=4 r-4-2 m r .
$$

To estimate $\left\|I_{1}\right\|_{L_{r}\left(\mathbb{D}^{4}\right)}$ we have to examine 
(3.14) $\int d x_{3} I_{1}^{r} \leq \int d x_{3} \int d x^{\prime} \int d t x_{3}^{4 r-4-2 m r} \frac{1}{t^{(5 / 2-m) r}} e^{-|x|^{2} /(4(\mu+\nu) t)} N^{r}(t)$

$$
\begin{aligned}
& =\int d x_{3} \int d x^{\prime} \int d t x_{3}^{4 r-4-2 m r} t^{(m-5 / 2) r+\alpha} e^{-|x|^{2} /(4(\mu+\nu) t)} \frac{N^{r}(t)}{t^{\alpha}} \\
& =\int d x_{3} \int d t x_{3}^{4 r-4-2 m r} t^{(m-5 / 2) r+\alpha+1} e^{-x_{3}^{2} /(4(\mu+\nu) t)} \frac{N^{r}(t)}{t^{\alpha}} .
\end{aligned}
$$

We consider

$$
J_{2}=\int d x_{3} x_{3}^{4 r-4-2 m r} t^{(m-5 / 2) r+\alpha+1} e^{-x_{3}^{2} /(4(\mu+\nu) t)} .
$$

Taking $w=x_{3} /(4(\mu+\nu) t)^{1 / 2}$ we get

$$
J_{2}=\int d w w^{4 r-2 m r-4} t^{1 / 2+2 r-m r-2+(m-5 / 2) r+\alpha+1} e^{-w^{2}} .
$$

$J_{2}$ is independent of $t$ if

$$
1 / 2+2 r-m r-2+(m-5 / 2) r+\alpha+1=0,
$$

which gives

$$
\alpha=\frac{1}{2}+\frac{1}{2} r
$$

and $J_{2}$ is finite if $4 r-2 m r-4>-1$ or

$$
m<2-\frac{3}{2 r} \text {. }
$$

From (3.12) and (3.16) we can find $m$. Then by (3.14) and (3.10),

$$
\int d x_{3} I_{1}^{r} \leq c \int d t \int d t^{\prime} \int d x^{\prime} \frac{\left|\phi\left(x^{\prime}, t^{\prime}-t\right)-\phi\left(x^{\prime}, t^{\prime}\right)\right|^{r}}{t^{\alpha}}
$$

and by (3.15) and (2.1) we get

$$
\left\|I_{1}\right\|_{L_{r}\left(\mathbb{D}^{4}\right)} \leq c\|\phi\|_{W_{r}^{0,1 / 2-1 /(2 r)\left(\mathbb{R}^{3}\right)}} .
$$

The case of $I_{2}$ is almost the same. Thus by Theorem 2.1 we obtain $\partial_{3} w \in$ $L_{r}\left(\mathbb{D}^{4}\right)$, which together with the results of [4] gives

$$
\|\nabla w\|_{L_{r}\left(\mathbb{D}^{4}\right)} \leq c\|\phi\|_{W_{r}^{1-1 / r, 1 / 2-1 /(2 r)}\left(\mathbb{R}^{3}\right)} .
$$

To finish the proof of Lemma 3.1 we have to consider problem (3.2) in the whole space

$$
\begin{aligned}
& w_{t}-(\mu+\nu) \Delta w=\operatorname{div} f, \\
& \left.w\right|_{t=0}=0,
\end{aligned}
$$

where supp $f \subset \mathbb{D}^{4}$. Applying the Fourier transform to (3.19) 1 gives

$$
r^{* 2} w=\sum_{k=1}^{3} i \xi_{k} f_{k},
$$


where $r^{*}=\sqrt{i \xi_{0} /(\mu+\nu)+|\xi|^{2}}$. Then we get

$$
w=\mathcal{F}_{t, x}^{-1}\left[\frac{\sum_{k=1}^{3} i \xi_{k} f_{k}}{r^{* 2}}\right]
$$

and by Theorem 2.1 we see that

$$
\|\nabla w\|_{L_{r}\left(\mathbb{R}^{4}\right)}+\left\|\mathcal{F}^{-1}\left|\xi_{0}\right|^{1 / 2} \mathcal{F} w\right\|_{L_{r}\left(\mathbb{R}^{4}\right)} \leq c\|f\|_{L_{r}\left(\mathbb{R}^{4}\right)} .
$$

From (3.20) we see that

$$
\left\|\varphi_{T} w\right\|_{H_{r}^{1,1 / 2}\left(\mathbb{R}^{4}\right)} \leq c(T)\|f\|_{L_{r}\left(\mathbb{R}^{4}\right)},
$$

where $\varphi_{T}(\cdot) \in C_{0}^{\infty}(\mathbb{R})$ and $\left.\varphi_{T}\right|_{[0, T]} \equiv 1$. Hence by Proposition 2.2 we get

$$
\|w\|_{W_{r}^{1,1 / 2}\left(\mathbb{R}^{3} \times[0, T]\right)} \leq c(T)\|f\|_{L_{r}\left(\mathbb{R}^{3} \times[0, T]\right)} .
$$

To solve (3.2) we consider the solution in the form

$$
w=w_{1}+w_{2},
$$

where $w_{2}$ is the solution of problem (3.19) and $w_{1}$ is the solution of problem (3.4) with $\phi=w_{0}-\left.w_{2}\right|_{x_{3}=0}$. From (3.18) and (3.22) we get (3.3).

LEMma 3.2. Let $f \in L_{r}\left(\mathbb{D}^{4}\right)$ and $g \in W_{r}^{2-1 / r, 1-1 /(2 r)}\left(\mathbb{R}^{3}\right)$. Then there exists a unique solution of the problem

$$
\begin{aligned}
& u_{t}-\mu \Delta u=f, \\
& \left.u\right|_{x_{3}=0}=g, \\
& \left.u\right|_{t=0}=0, \\
& u \rightarrow 0 \quad \text { as } x_{3} \rightarrow \infty,
\end{aligned}
$$

such that $u \in W_{r}^{2,1}\left(\mathbb{D}_{T}^{4}\right)$ and the following estimate holds:

$$
\begin{aligned}
\left\|D_{t} u\right\|_{L_{r}\left(\mathbb{D}_{T}^{4}\right)}+\left\|D_{x}^{2} u\right\|_{L_{r}\left(\mathbb{D}_{T}^{4}\right)} & \\
& \leq c(T)\left(\|f\|_{L_{r}\left(\mathbb{D}_{T}^{4}\right)}+\|g\|_{W_{r}^{2-1 / r, 1-1 /(2 r)}\left(\mathbb{R}_{T}^{3}\right)}\right) .
\end{aligned}
$$

Proof. The proof is an easy consequence of the proof of Lemma 3.1.

Using Lemmas 3.1 and 3.2 we show the following result.

LEMmA 3.3. Let $g \in W_{r}^{2-1 / r, 1-1 /(2 r)}\left(\mathbb{R}^{3}\right)$. Then there exists a unique solution of the problem

$$
\begin{aligned}
& v_{t}-\mu \Delta v-\nu \nabla \operatorname{div} v+a \nabla \eta=0 \\
& \eta_{t}+b \operatorname{div} v=0 \\
& \left.v\right|_{x_{3}=0}=g, \\
& \left.v\right|_{t=0}=0,\left.\quad \eta\right|_{t=0}=0 \\
& v, \eta \rightarrow 0 \quad \text { as } x_{3} \rightarrow \infty
\end{aligned}
$$


such that $v \in W_{r}^{2,1}\left(\mathbb{D}_{T}^{4}\right)$ and $\nabla \eta, \nabla \eta_{t} \in L_{r}\left(\mathbb{D}_{T}^{4}\right)$ and the following estimate holds:

$$
\begin{aligned}
\left\|D_{t} v\right\|_{L_{r}\left(\mathbb{D}_{T}^{4}\right)}+\left\|D_{x}^{2} v\right\|_{L_{r}\left(\mathbb{D}_{T}^{4}\right)}+\|\nabla \eta\|_{L_{r}\left(\mathbb{D}_{T}^{4}\right)}+\left\|\nabla \eta_{t}\right\|_{L_{r}\left(\mathbb{D}_{T}^{4}\right)} \\
\leq c(T)\|g\|_{W_{r}^{2-1 / r, 1-1 /(2 r)}\left(\mathbb{R}_{T}^{3}\right)}
\end{aligned}
$$

where $\mathbb{D}_{T}^{4}=\mathbb{R}_{x^{\prime}}^{2} \times[0, \infty)_{x_{3}} \times[0, T]_{t}$.

Proof. To solve (3.26) we apply the Fourier-Laplace transform

$$
\begin{aligned}
& \mathcal{L}_{\gamma}[v]=u\left(s, \xi^{\prime}, x_{3}\right)=\int e^{-s t} \int e^{-i \xi^{\prime} x^{\prime}} u\left(t, x^{\prime}, x_{3}\right) d t d x^{\prime}, \\
& \mathcal{L}_{\gamma}[\eta]=q\left(s, \xi^{\prime}, x_{3}\right)=\int e^{-s t} \int e^{-i \xi^{\prime} x^{\prime}} \eta\left(t, x^{\prime}, x_{3}\right) d t d x^{\prime},
\end{aligned}
$$

where $s=\gamma+i \xi_{0}$ with $\gamma>2 a b /(\mu+\nu)$.

Note. The above transformation can be treated as the Fourier transform because we are interested in the results which are local in time. Thus we will be able to use the relation

$$
x(t)=\mathcal{L}_{\gamma}^{-1} \mathcal{L}_{\gamma}[x(t)]=e^{\gamma t} \mathcal{F}^{-1} \mathcal{F}\left[e^{-\gamma t} x(t)\right] .
$$

After taking the Fourier-Laplace transform, $(3.26)_{1,2}$ reads

$$
\begin{aligned}
& s u_{1}-\mu\left[-\left|\xi^{\prime}\right|^{2} u_{1}+u_{1,33}\right]-\nu i \xi_{1}\left[i \xi_{1} u_{1}+i \xi_{2} u_{2}+u_{3,3}\right]+i a \xi_{1} q=0, \\
& s u_{2}-\mu\left[-\left|\xi^{\prime}\right|^{2} u_{2}+u_{2,33}\right]-\nu i \xi_{2}\left[i \xi_{1} u_{1}+i \xi_{2} u_{2}+u_{3,3}\right]+i a \xi_{2} q=0, \\
& s u_{3}-\mu\left[-\left|\xi^{\prime}\right|^{2} u_{3}+u_{3,33}\right]-\nu\left[i \xi_{1} u_{1}+i \xi_{2} u_{2}+u_{3,3}\right]_{, 3}+a q_{, 3}=0, \\
& s q+b i \xi_{1} u_{1}+b i \xi_{2} u_{2}+b u_{3,3}=0
\end{aligned}
$$

and after some calculations we get (3.26) in the form

$$
\begin{aligned}
& u_{1,33}=r^{2} u_{1}+i \xi_{1} \alpha q, \\
& u_{2,33}=r^{2} u_{2}+i \xi_{2} \alpha q, \\
& u_{3,3}=-i \xi_{1} u_{1}-i \xi_{2} u_{2}-\frac{s}{b} q, \\
& q_{, 3}=-\beta r^{2} u_{3}-\beta i \xi_{1} u_{1,3}-\beta i \xi_{2} u_{2,3}, \\
& \left.u\right|_{x_{3}=0}=g, \\
& u, q \rightarrow 0 \text { as } x_{3} \rightarrow \infty,
\end{aligned}
$$

where

$$
\begin{gathered}
r^{2}=s / \mu+\left|\xi^{\prime}\right|^{2}, \quad \arg r \in(-\pi / 4, \pi / 4), \\
\alpha=\frac{\nu s+a b}{\mu b}, \quad \beta=\frac{b \mu}{(\mu+\nu) s+a b} .
\end{gathered}
$$


Solving (3.28) we get

$$
\begin{aligned}
\left(\begin{array}{l}
u_{1} \\
u_{2} \\
u_{3}
\end{array}\right)= & \phi_{1}\left(\begin{array}{c}
0 \\
-1 / r \\
-i \xi_{2} / r^{2}
\end{array}\right) e^{-r x_{3}}+\phi_{2}\left(\begin{array}{c}
-1 / r \\
0 \\
-i \xi_{1} / r^{2}
\end{array}\right) e^{-r x_{3}} \\
& +\phi_{3}\left(\begin{array}{c}
-\xi_{1} / \lambda_{3} \\
-\xi_{2} / \lambda_{3} \\
-i
\end{array}\right) e^{-\lambda_{3} x_{3}} \\
q= & \phi_{3} \frac{i\left(-r^{2}+\lambda_{3}^{2}\right)}{\lambda_{3}} e^{-\lambda_{3} x_{3}}
\end{aligned}
$$

where

$$
\lambda_{3}=\sqrt{\frac{s^{2}}{(\mu+\nu) s+a b}+\left|\xi^{\prime}\right|^{2}}, \quad \arg \lambda_{3} \in(-\pi / 4, \pi / 4) .
$$

To compute $\phi_{i}$ we use the boundary condition $(3.28)_{5}$

$$
\left(\begin{array}{l}
g_{1} \\
g_{2} \\
g_{3}
\end{array}\right)=\left(\begin{array}{ccc}
0 & -1 / r & -\xi_{1} / \lambda_{3} \\
-1 / r & 0 & -\xi_{2} / \lambda_{3} \\
-i \xi_{2} / r^{2} & -i \xi_{1} / r^{2} & -i
\end{array}\right)\left(\begin{array}{c}
\phi_{1} \\
\phi_{2} \\
\phi_{3}
\end{array}\right) .
$$

From (3.30) we get

$$
\left(\begin{array}{c}
\phi_{1} \\
\phi_{2} \\
\phi_{3}
\end{array}\right)=\left(\begin{array}{ccc}
\frac{r \xi_{1} \xi_{2}}{-\lambda_{3} r+\left|\xi^{\prime}\right|^{2}} & \frac{r\left(\lambda_{3} r-\xi_{1}^{2}\right)}{-\lambda_{3} r+\left|\xi^{\prime}\right|^{2}} & \frac{i r^{2} \xi_{2}}{-\lambda_{3} r+\left|\xi^{\prime}\right|^{2}} \\
\frac{r\left(\lambda_{3} r-\xi_{2}^{2}\right)}{-\lambda_{3} r+\left|\xi^{\prime}\right|^{2}} & \frac{r \xi_{1} \xi_{2}}{-\lambda_{3} r+\left|\xi^{\prime}\right|^{2}} & \frac{i r^{2} \xi_{1}}{-\lambda_{3} r+\left|\xi^{\prime}\right|^{2}} \\
\frac{-\lambda_{3} \xi_{1}}{-\lambda_{3} r+\left|\xi^{\prime}\right|^{2}} & \frac{-\lambda_{3} \xi_{2}}{-\lambda_{3} r+\left|\xi^{\prime}\right|^{2}} & \frac{-i \lambda_{3} r}{-\lambda_{3} r+\left|\xi^{\prime}\right|^{2}}
\end{array}\right)\left(\begin{array}{l}
g_{1} \\
g_{2} \\
g_{3}
\end{array}\right)
$$

Applying $(3.29)_{2}$ we get

$$
\begin{aligned}
q= & \left(\frac{-\lambda_{3} \xi_{1}}{-\lambda_{3} r+\left|\xi^{\prime}\right|^{2}} g_{1}+\frac{-\lambda_{3} \xi_{2}}{-\lambda_{3} r+\left|\xi^{\prime}\right|^{2}} g_{2}+\frac{-i \lambda_{3} r}{-\lambda_{3} r+\left|\xi^{\prime}\right|^{2}} g_{3}\right) \\
& \times \frac{i\left(-r^{2}+\lambda_{3}^{2}\right)}{\lambda_{3}} e^{-\lambda_{3} x_{3}} .
\end{aligned}
$$

Now we assume that $g_{1}=g_{2}=0$ (other cases are similar). Then

$$
q=\frac{-i \lambda_{3} r}{-\lambda_{3} r+\left|\xi^{\prime}\right|^{2}} g_{3} \frac{i\left(-r^{2}+\lambda_{3}^{2}\right)}{\lambda_{3}} e^{-\lambda_{3} x_{3}} \text {. }
$$

Since

$$
\left|\xi_{0}^{j_{1}} \xi_{1}^{j_{2}} \xi_{2}^{j_{3}} \partial_{\xi_{0}}^{j_{1}} \partial_{\xi_{1}}^{j_{2}} \partial_{\xi_{2}}^{j_{3}}\left(\frac{\left(-r^{2}+\lambda_{3}^{2}\right)}{-\lambda_{3} r+\left|\xi^{\prime}\right|^{2}} \cdot \frac{r}{i \xi_{1}+\xi_{2}}\right)\right| \leq c,
$$

where $j_{k}=0$ or 1 , and (with $\left.r^{\prime}=\sqrt{i \xi_{0} /(\mu+\nu)+\left|\xi^{\prime}\right|^{2}}\right)$

$$
\left|\frac{e^{-\lambda_{3} x_{3}}}{e^{-r^{\prime} x_{3}}}\right| \leq c
$$


which follows from $\arg \left(\lambda_{3}-r^{\prime}\right) \in(-\pi / 2, \pi / 2)$ and $\operatorname{Re}\left(\lambda_{3}-r^{\prime}\right)>0$ with $\gamma>2 a b /(\mu+\nu)$, and since we also have

$$
\left|s^{j_{1}} \xi_{1}^{j_{2}} \xi_{2}^{j_{3}} \partial_{s}^{j_{1}} \partial_{\xi_{1}}^{j_{2}} \partial_{\xi_{2}}^{j_{3}}\left(\lambda_{3}-r^{\prime}\right)\right| \leq c
$$

where $j_{k}=0$ or 1 , it follows that

$$
\begin{aligned}
q & =\frac{r^{2}+\lambda_{3}^{2}}{-\lambda_{3} r+\left|\xi^{\prime}\right|^{2}} \cdot \frac{r}{i \xi_{1}+\xi_{2}} e^{-x_{3}\left(\lambda_{3}-r^{\prime}\right)}\left(i \xi_{1}+\xi_{2}\right) g_{3} e^{-r^{\prime} x_{3}} \\
& =\Xi\left(i \xi_{1}+\xi_{2}\right) g_{3} e^{-r^{\prime} x_{3}} .
\end{aligned}
$$

Obviously $\left(i \xi_{1}+\xi_{2}\right) g_{3} \in W_{r}^{1-1 / r, 1 / 2-1 /(2 r)}$. Thus by (3.33)-(3.35) we see that $\Xi$ satisfies the conditions for multipliers, and by Theorem 2.1 we get

$$
D_{x^{\prime}} \eta \in L_{r}\left(\mathbb{D}^{4}\right) \text {. }
$$

We have to show the regularity in $x_{3}$. We have

$$
\begin{aligned}
\partial_{x_{3}} q & =\frac{r^{2}+\lambda_{3}^{2}}{-\lambda_{3} r+\left|\xi^{\prime}\right|^{2}} r \lambda_{3} e^{-\lambda_{3} x_{3}} g_{3} \\
& =\frac{r^{2}+\lambda_{3}^{2}}{-\lambda_{3} r+\left|\xi^{\prime}\right|^{2}} r\left(\lambda_{3}-r^{\prime}\right) e^{-\lambda_{3} x_{3}} g_{3}+\frac{r^{2}+\lambda_{3}^{2}}{-\lambda_{3} r+\left|\xi^{\prime}\right|^{2}} r r^{\prime} e^{-\lambda_{3} x_{3}} g_{3} \\
& =G_{1}+G_{2} .
\end{aligned}
$$

$G_{1}$ can be estimated in the same way as for the $x^{\prime}$ regularity. So we treat only $G_{2}$ :

$$
G_{2}=\frac{r^{2}+\lambda_{3}^{2}}{-\lambda_{3} r+\left|\xi^{\prime}\right|^{2}} \cdot \frac{r r^{\prime}}{s+\left|\xi^{\prime}\right|^{2}} e^{-\left(\lambda_{3}-r^{\prime}\right) x_{3}}\left(s+\left|\xi^{\prime}\right|^{2}\right) e^{-r^{\prime} x_{3}} g_{3}
$$

and one can see that

$$
\left|\xi_{0}^{j_{1}} \xi_{1}^{j_{2}} \xi_{2}^{j_{3}} \partial_{\xi_{0}}^{j_{1}} \partial_{\xi_{1}}^{j_{2}} \partial_{\xi_{2}}^{j_{3}}\left(\frac{r^{2}+\lambda_{3}^{2}}{-\lambda_{3} r+\left|\xi^{\prime}\right|^{2}} \cdot \frac{r r^{\prime}}{s+\left|\xi^{\prime}\right|^{2}} e^{-\left(\lambda_{3}-r^{\prime}\right) x_{3}}\right)\right| \leq c
$$

where $j_{k}=0$ or 1 . So by Theorem 2.1 we have $G_{2} \in L_{r}$ if $\mathcal{F}^{-1}\left[s e^{-r^{\prime} x_{3}} g_{3}\right]$ $\in L_{r}$; but repeating (3.7)-(3.17) for $I$ we get

$$
\left\|\mathcal{F}^{-1}\left[s e^{-r^{\prime} x_{3}} g_{3}\right]\right\|_{L_{r}}=\left\|\Gamma_{, t x_{3}} * g_{3}\right\|_{L_{r}} \leq c\left\|g_{3}\right\|_{W_{r}^{2-1 / r, 1-1 /(2 r)}} .
$$

Thus we get $q \in W_{r}^{1,0}$ and $\eta \in W_{r(\text { loc })}^{1,0}\left(\mathbb{D}^{4}\right)$ and

$$
\|\nabla \eta\|_{L_{r}\left(\mathbb{D}^{4}\right)} \leq c\|g\|_{W_{r}^{2-1 / r, 1-1 /(2 r)}\left(\mathbb{R}^{3}\right)} .
$$

And from (3.29)-(3.31) we get

$$
\left.\nabla v\right|_{x_{3}=0} \in W_{r}^{1-1 / r, 1 / 2-1 /(2 r)}\left(\mathbb{R}^{3}\right)
$$

and

$$
\left\|\left.\nabla v\right|_{x_{3}=0}\right\|_{W_{r}^{1-1 / r, 1 / 2-1 /(2 r)}\left(\mathbb{R}^{3}\right)} \leq c\|g\|_{W_{r}^{2-1 / r, 1-1 /(2 r)}\left(\mathbb{R}^{3}\right)} .
$$


We show this for one term:

$$
\begin{aligned}
\left.\partial_{3} u_{3}\right|_{x_{3}=0} & =\phi_{1} \frac{i \xi_{2}}{r}+\phi_{2} \frac{i \xi_{1}}{r}+\phi_{3} i \lambda_{3} \\
& =\frac{i \xi_{1} \lambda_{3}\left(r-\lambda_{3}\right)}{-\lambda_{3} r+\left|\xi^{\prime}\right|^{2}} g_{1}+\frac{i \xi_{2} \lambda_{3}\left(r-\lambda_{3}\right)}{-\lambda_{3} r+\left|\xi^{\prime}\right|^{2}} g_{2}+\frac{-r\left(\left|\xi^{\prime}\right|^{2}-\lambda_{3}^{2}\right)}{-\lambda_{3} r+\left|\xi^{\prime}\right|^{2}} g_{3} \\
& =\left.\left(U_{, 3}^{1}+U_{, 3}^{2}+U_{, 3}^{3}\right)\right|_{x_{3}=0},
\end{aligned}
$$

where

$$
\begin{aligned}
U^{1} & =-\frac{i \xi_{1} \lambda_{3}\left(r-\lambda_{3}\right)}{r\left(-\lambda_{3} r+\left|\xi^{\prime}\right|^{2}\right)} g_{1} e^{-r x_{3}}, \\
U^{2} & =-\frac{i \xi_{2} \lambda_{3}\left(r-\lambda_{3}\right)}{\left(-\lambda_{3} r+\left|\xi^{\prime}\right|^{2}\right) r} g_{2} e^{-r x_{3}}, \\
U^{3} & =-\frac{-\left(\left|\xi^{\prime}\right|^{2}-\lambda_{3}^{2}\right)}{-\lambda_{3} r+\left|\xi^{\prime}\right|^{2}} g_{3} e^{-r x_{3}} .
\end{aligned}
$$

We need to have $U^{i} \in W_{r}^{2,1}$. From (3.33) we see that it is enough to have $\mathcal{F}_{t, x^{\prime}}^{-1}\left[g_{i} e^{-r x_{3}}\right] \in W_{r}^{2,1}$, which follows from (3.5), (3.17) and (3.39); hence

$$
\left\|U^{i}\right\|_{W_{r}^{2,1}} \leq c\left\|g_{i}\right\|_{W_{r}^{2-1 / r, 1-1 /(2 r)}}
$$

and from the trace theorem we get (3.41).

Since $\eta \in W_{r(\text { loc })}^{1,0}\left(\mathbb{D}^{4}\right)$ (see $\left.(3.40)\right)$ problem $(3.26)$ reduces to

$$
\begin{aligned}
& v_{t}-\mu \Delta v-\nu \nabla \operatorname{div} v=f^{\prime}, \\
& \left.v\right|_{x_{3}=0}=g, \\
& \left.v\right|_{t=0}=0, \\
& v \rightarrow 0 \quad \text { as } x_{3} \rightarrow \infty
\end{aligned}
$$

where $f^{\prime}=-a \nabla \eta \in L_{r}\left(\mathbb{D}_{T}^{4}\right)$ and $g \in W_{r}^{2-2 / r, 1-1 /(2 r)}\left(\mathbb{R}_{T}^{3}\right)$.

Taking divergence of $(3.44)_{1}$ and setting $w=\operatorname{div} v$ we get

$$
\begin{aligned}
& w_{t}-(\mu+\nu) \Delta w=\operatorname{div} f^{\prime}, \\
& \left.w\right|_{x_{3}=0}=w_{0}, \\
& \left.w\right|_{t=0}=0, \\
& w \rightarrow 0 \quad \text { as } x_{3} \rightarrow \infty,
\end{aligned}
$$

where $f^{\prime} \in L_{r}\left(\mathbb{D}_{T}^{4}\right)$ and by (3.41) we have

$$
w_{0}=\left.\operatorname{div} v\right|_{x_{3}=0} \in W_{r}^{1-1 / r, 1 / 2-1 /(2 r)}\left(\mathbb{R}_{T}^{3}\right) .
$$

Applying Lemma 3.1 we get $\operatorname{div} v \in W_{r}^{1,0}\left(\mathbb{D}_{T}^{4}\right)$ and

$$
\|\nabla v\|_{L_{r}\left(\mathbb{D}_{T}^{4}\right)} \leq c(T)\left(\|f\|_{L_{r}\left(\mathbb{D}_{T}^{4}\right)}+\left\|w_{0}\right\|_{W_{r}^{1-1 / r, 1 / 2-1 /(2 r)}\left(\mathbb{R}_{T}^{3}\right)}\right) .
$$


Thus we reduce problem (3.44) to

$$
\begin{aligned}
& v_{t}-\mu \Delta v=f^{\prime \prime}, \\
& \left.v\right|_{x_{3}=0}=g, \\
& \left.v\right|_{t=0}=0, \\
& v \rightarrow 0 \quad \text { as } x_{3} \rightarrow \infty,
\end{aligned}
$$

where $f^{\prime \prime}=-a \nabla \eta+\nu \nabla \operatorname{div} v \in L_{r}\left(\mathbb{D}_{T}^{4}\right)$. Now by Lemma 3.2 we get from problem (3.47) a solution of problem (3.26) and from (3.26) 2 we obtain $\nabla \eta_{t} \in L_{r}\left(\mathbb{D}_{T}^{4}\right)$ and we show (3.27). Hence the proof of Lemma 3.3 is finished.

Next we consider (3.1) in the whole space:

$$
\begin{aligned}
& u_{t}-\mu \Delta u-\nu \nabla \operatorname{div} u+a \nabla \eta=f, \\
& \eta_{t}+b \operatorname{div} u=h, \\
& \left.u\right|_{t=0}=0,\left.\quad \eta\right|_{t=0}=0 .
\end{aligned}
$$

LEMmA 3.4. Let $f \in L_{r}\left(\mathbb{D}^{4}\right)$ and $h \in W_{r}^{1,0}\left(\mathbb{D}^{4}\right)$. Then problem $(3.48)$ has a unique solution $u \in W_{r(\text { loc })}^{2,1}\left(\mathbb{R}^{4}\right), \eta_{t x} \in L_{r}\left(\mathbb{R}^{4}\right)$ and the following estimate holds:

$$
\begin{aligned}
& \left\|D_{t} u\right\|_{L_{r}\left(\mathbb{R}^{3} \times(0, T)\right)}+\left\|D_{x}^{2} u\right\|_{L_{r}\left(\mathbb{R}^{3} \times(0, T)\right)}+\left\|D_{x} \eta\right\|_{L_{r}\left(\mathbb{R}^{3} \times(0, T)\right)} \\
& \quad+\left\|D_{t} D_{x} \eta\right\|_{L_{r}\left(\mathbb{R}^{3} \times(0, T)\right)} \leq c\left(\|f\|_{L_{r}\left(\mathbb{R}^{3} \times(0, T)\right)}+\|h\|_{W_{r}^{1,0}\left(\mathbb{R}^{3} \times(0, T)\right)}\right) .
\end{aligned}
$$

To solve (3.48) we apply the Fourier transform

$$
\begin{aligned}
& \mathcal{F}[u]=v(s, \xi)=\int e^{-s t} \int e^{-i \xi x} u(t, x) d x d t \\
& \mathcal{F}[\eta]=q(s, \xi)=\int e^{-s t} \int e^{-i \xi x} \eta(t, x) d x d t
\end{aligned}
$$

where $s=i \xi_{0}$. In these terms (3.48) reads

$$
\begin{aligned}
& \left(s+\mu|\xi|^{2}+\nu \xi_{1}^{2}\right) v_{1}+\nu \xi_{1} \xi_{2} v_{2}+\nu \xi_{1} \xi_{3} v_{3}+i a \xi_{1} q=f_{1} \\
& \nu \xi_{2} \xi_{1} v_{1}+\left(s+\mu|\xi|^{2}+\nu \xi_{2}^{2}\right) v_{2}+\nu \xi_{2} \xi_{3} v_{3}+i a \xi_{2} q=f_{2} \\
& \nu \xi_{3} \xi_{1} v_{1}+\nu \xi_{3} \xi_{2} v_{2}+\left(s+\mu|\xi|^{2}+\nu \xi_{3}^{2}\right) v_{3}+i a \xi_{3} q=f_{3} \\
& i b \xi_{1} v_{1}+i b \xi_{2} v_{2}+i b \xi_{3} v_{3}+s q=g
\end{aligned}
$$

or

$$
A\left[\begin{array}{c}
v_{1} \\
v_{2} \\
v_{3} \\
q
\end{array}\right]=\left[\begin{array}{c}
f_{1} \\
f_{2} \\
f_{3} \\
h
\end{array}\right],
$$


where

$$
A=\left[\begin{array}{cccc}
s+\mu|\xi|^{2}+\nu \xi_{1}^{2} & \nu \xi_{1} \xi_{2} & \nu \xi_{1} \xi_{3} & i a \xi_{1} \\
\nu \xi_{2} \xi_{1} & s+\mu|\xi|^{2}+\nu \xi_{2}^{2} & \nu \xi_{2} \xi_{3} & i a \xi_{2} \\
\nu \xi_{2} \xi_{1} & \nu \xi_{3} \xi_{2} & s+\mu|\xi|^{2}+\nu \xi_{3}^{2} & i a \xi_{3} \\
i b \xi_{1} & i b \xi_{2} & i b \xi_{3} & s
\end{array}\right]
$$

From (3.51) we express the solution by the formula

$$
\left[\begin{array}{c}
v_{1} \\
v_{2} \\
v_{3} \\
q
\end{array}\right]=A^{-1}\left[\begin{array}{c}
f_{1} \\
f_{2} \\
f_{3} \\
h
\end{array}\right],
$$

where $A^{-1}$ is defined by (here $r^{2}=s+\mu|\xi|^{2}$ )

$$
\left[\begin{array}{cccc}
\frac{r^{2} s+(\nu s+a b)\left(\xi_{2}^{2}+\xi_{3}^{2}\right)}{r^{2}\left(r^{2} s+(\nu s+a b)|\xi|^{2}\right)} & \frac{-(\nu s+a b) \xi_{1} \xi_{2}}{r^{2}\left(r^{2} s+(\nu s+a b)|\xi|^{2}\right)} & \frac{-(\nu s+a b) \xi_{1} \xi_{3}}{r^{2}\left(r^{2} s+(\nu s+a b)|\xi|^{2}\right)} & \frac{-i a \xi_{1}}{r^{2} s+(\nu s+a b)|\xi|^{2}} \\
\frac{-(\nu s+a b) \xi_{2} \xi_{1}}{r^{2}\left(r^{2} s+(\nu s+a b)|\xi|^{2}\right)} & \frac{r^{2} s+(\nu s+a b)\left(\xi_{1}^{2}+\xi_{3}^{2}\right)}{r^{2}\left(r^{2} s+(\nu s+a b)|\xi|^{2}\right)} & \frac{-(\nu s+a b) \xi_{2} \xi_{3}}{r^{2}\left(r^{2} s+(\nu s+a b)|\xi|^{2}\right)} & \frac{-a \xi_{2}}{r^{2} s+(\nu s+a b)|\xi|^{2}} \\
\frac{-(\nu s+a b) \xi_{1} \xi_{3}}{r^{2}\left(r^{2} s+(\nu s+a b)|\xi|^{2}\right)} & \frac{-(\nu s+a b) \xi_{3} \xi_{2}}{r^{2}\left(r^{2} s+(\nu s+a b)|\xi|^{2}\right)} & \frac{r^{2} s+(\nu s+a b)\left(\xi_{1}^{2}+\xi_{2}^{2}\right)}{r^{2}\left(r^{2} s+(\nu s+a b)|\xi|^{2}\right)} & \frac{-i a \xi_{3}}{r^{2} s+(\nu s+a b)|\xi|^{2}} \\
\frac{-i b \xi_{1}}{r^{2} s+(\nu s+a b)|\xi|^{2}} & \frac{-i b \xi_{2}}{r^{2} s+(\nu s+a b)|\xi|^{2}} & \frac{-i b \xi_{3}}{r^{2} s+(\nu s+a b)|\xi|^{2}} & \frac{r^{2}+\nu|\xi|^{2}}{r^{2} s+(\nu s+a b)|\xi|^{2}}
\end{array}\right]
$$

One can check that

$$
\left.\left.\left|r^{2} s+(\nu s+a b)\right| \xi\right|^{2}|\geq c| \xi_{0}|\cdot| \xi\right|^{2} .
$$

Using (3.52) and (3.53) we can reduce (3.48) to a parabolic system and easily get (3.49).

Lemmas 3.3 and 3.4 gives the result of this section.

LEMmA 3.5. Let $f \in L_{r}\left(\mathbb{D}^{4}\right), h \in W_{r}^{1,0}\left(\mathbb{D}^{4}\right), g \in W_{r}^{2-1 / r, 1-1 /(2 r)}\left(\mathbb{R}^{3}\right)$. Then problem (3.1) has a unique solution $u \in W_{r(\text { loc })}^{2,1}\left(\mathbb{D}^{4}\right), \eta_{t x} \in L_{r}\left(\mathbb{D}^{4}\right)$ and the following estimate holds:

$$
\begin{aligned}
& \left\|D_{t} u\right\|_{L_{r}\left(\mathbb{D}_{T}^{4}\right)}+\left\|D_{x}^{2} u\right\|_{L_{r}\left(\mathbb{D}_{T}^{4}\right)}+\left\|D_{x} \eta\right\|_{L_{r}\left(\mathbb{D}_{T}^{4}\right)}+\left\|D_{t} D_{x} \eta\right\|_{L_{r}\left(\mathbb{D}_{T}^{4}\right)} \\
& \quad \leq c(T)\left(\|f\|_{L_{r}\left(\mathbb{D}_{T}^{4}\right)}+\|h\|_{W_{r}^{1,0}\left(\mathbb{D}_{T}^{4}\right)}+\|g\|_{W_{r}^{2-1 / r, 1-1 /(2 r)}\left(\mathbb{R}_{T}^{3}\right)}\right) .
\end{aligned}
$$

4. Problem in a bounded domain. To solve problem (1.1) in a bounded domain $\Omega$ we have to define a partition of unity. We take two collections of open sets: $\left\{\omega^{(k)}\right\}$ and $\left\{\Omega^{(k)}\right\}$ such that $\overline{\omega^{(k)}} \subset \Omega^{(k)} \subset \Omega$, $\bigcup_{k} \omega^{(k)}=\Omega$ and $\bigcup_{k} \Omega^{(k)}=\Omega$ with $k \in \mathcal{M} \cup \mathcal{N}$ where if $k \in \mathcal{M}$ then $\overline{\Omega^{(k)}} \cap S=\emptyset$ and if $k \in \mathcal{N}$ then $\overline{\omega^{(k)}} \cap S \neq \emptyset$.

We assume that

$$
\sup _{k} \operatorname{diam} \Omega^{(k)} \leq 2 \lambda
$$


for some $\lambda$ small enough. Let $\zeta^{(k)}$ be a smooth function such that $0 \leq$ $\zeta^{(k)} \leq 1$ and $\zeta^{(k)}(x)=1$ for $x \in \omega^{(k)}, \zeta^{(k)}(x)=0$ for $x \in \Omega \backslash \Omega^{(k)}$ and $\left|D_{x}^{\nu} \zeta^{(k)}(x)\right| \leq c / \lambda^{|\nu|}$ and $1 \leq \sum_{k}\left(\zeta^{(k)}\right)^{2} \leq N_{0}$.

Let

$$
\pi^{(k)}=\frac{\zeta^{(k)}}{\sum_{l}\left(\zeta^{(l)}\right)^{2}} .
$$

It is easy to see that $\pi^{(k)}=0$ for $x \in \Omega \backslash \Omega^{(k)},\left|D_{x}^{\nu} \pi^{(k)}(x)\right| \leq c / \lambda^{|\nu|}$ and

$$
\sum_{k} \pi^{(k)} \zeta^{(k)}=1
$$

We denote by $\xi^{(k)}$ a fixed point of $\omega^{(k)}$ for $k \in \mathcal{M}$, and of $\omega^{(k)} \cap S$ for $k \in \mathcal{N}$.

Let us consider a local coordinate system $y=\left(y_{1}, y_{2}, y_{3}\right)$ with center at $\xi^{(k)}$. If $k \in \mathcal{N}$ then the boundary part $\widetilde{S^{(k)}}=S \cap \overline{\Omega^{(k)}}$ is described by $y_{3}=F\left(y_{1}, y_{2}\right)$. We choose the local system such that $F(0)=0$ and $\nabla F(0)=0$. From $S \in W_{r}^{2-1 / r}$ we have $F \in W_{r}^{2-1 / r}$. Extend $F$ to $\bar{F}$ in such a way that

$$
\bar{F}\left(y_{1}, y_{2}, 0\right)=F\left(y_{1}, y_{2}\right) \quad \text { and } \quad \bar{F} \in W_{r}^{2}
$$

as well as

$$
\bar{F} \in C^{1+\alpha} \quad \text { with } \quad 0<\alpha<1-3 / r \quad \text { and } \quad|\nabla \bar{F}| \leq c \lambda^{\alpha} .
$$

Now we can transform $\Omega^{(k)}$ into the halfspace by

$$
z=\Phi_{k}(y)=(\operatorname{Id}-\bar{F})(y) .
$$

Let $y=Y_{k}(x)$ be a transformation to the local coordinates $y$ which consists of translations and rotations.

We introduce the following notation:

$$
L\left(x, t ; \partial_{x}\right)(u, \eta)=\left(\begin{array}{l}
L_{1}\left(x, t ; \partial_{x}\right)(u, \eta) \\
L_{2}\left(x, t ; \partial_{x}\right)(u, \eta)
\end{array}\right)
$$

where

$$
\left(\begin{array}{l}
L_{1}\left(x, t ; \partial_{x}\right)(u, \eta) \\
L_{2}\left(x, t ; \partial_{x}\right)(u, \eta)
\end{array}\right)=\left(\begin{array}{c}
C(x, t) u_{t}-\mu \Delta u-\nu \nabla \operatorname{div} u+A(x, t) \nabla \eta \\
\eta_{t}+B(x, t) \operatorname{div} u
\end{array}\right) .
$$

In this section we obtain the existence for the following problem:

$$
\begin{aligned}
& L\left(x, t ; \partial_{x}\right)(u, \eta)=(f, h)^{T}, \\
& \left.u\right|_{S_{T}}=g, \\
& \left.u\right|_{t=0}=0,\left.\quad \eta\right|_{t=0}=0 .
\end{aligned}
$$

Define $f^{(k)}(x, t)=\zeta^{(k)} f(x, t), h^{(k)}(x, t)=\zeta^{(k)} h(x, t)$ and $g^{(k)}(x, t)=$ $\zeta^{(k)} g(x, t)$.

For $k \in \mathcal{M}$ we define

$$
\left(u^{(k)}, \eta^{(k)}\right)(x, t)=R^{(k)}\left(f^{(k)}(x, t), h^{(k)}(x, t)\right)
$$


where $\left(u^{(k)}, \eta^{(k)}\right)(x, t)$ is a solution of the Cauchy problem

$$
L\left(\xi^{(k)}, 0 ; \partial_{x}\right)\left(u^{(k)}, \eta^{(k)}\right)=\left(f^{(k)}, h^{(k)}\right)^{T} .
$$

For $k \in \mathcal{N}$ we define

$$
\left(\widehat{u}^{(k)}, \widehat{\eta}^{(k)}\right)(z, t)=R^{(k)}\left(\widehat{f}^{(k)}(z, t), \widehat{h}^{(k)}(z, t) ; \widehat{g}^{(k)}(z, t)\right),
$$

where $\left(\widehat{u}^{(k)}, \widehat{\eta}^{(k)}\right)(z, t)=Z^{-1}\left(u^{(k)}, \eta^{(k)}\right)(x, t)$ is a solution of the problem

$$
\begin{aligned}
& L\left(\widehat{\xi}^{(k)}, 0 ; \partial_{z}\right)\left(\widehat{u}^{(k)}, \widehat{\eta}^{(k)}\right)=\left(\widehat{f}^{(k)}, \widehat{h}^{(k)}\right)^{T}, \\
& \left.\widehat{u}^{(k)}\right|_{z_{3}=0}=\widehat{g}^{(k)} .
\end{aligned}
$$

Now we can define the operator $R$, called the regularizer, by

$$
R \bar{d}=\left(\sum_{k \in \mathcal{M} \cup \mathcal{N}} \pi^{(k)}(x) u^{(k)}(x, t), \sum_{k \in \mathcal{M} \cup \mathcal{N}} \pi^{(k)} \eta^{(k)}(x) \eta^{(k)}(x, t)\right),
$$

where

$$
\bar{d}^{(k)}= \begin{cases}\left(f^{(k)}(x, t), h^{(k)}(x, t)\right)^{T}, & k \in \mathcal{M}, \\ Z_{k}\left[\left(\widehat{f}^{(k)}(z, t), \widehat{h}^{(k)}(z, t) ; \widehat{g}^{(k)}(z, t)\right)^{T}\right], & k \in \mathcal{N}\end{cases}
$$

and

$$
\left(u^{(k)}, \eta^{(k)}\right)(x, t)= \begin{cases}R^{(k)}\left(f^{(k)}(x, t), h^{(k)}(x, t)\right), & k \in \mathcal{M}, \\ Z_{k} R^{(k)}\left[\left(f^{(k)}(z, t), h^{(k)}(z, t) ; g^{(k)}(z, t)\right)\right], & k \in \mathcal{N},\end{cases}
$$

Let

$$
\beta=\lambda^{\alpha}+\frac{T^{1 / r}+T^{1 / 2}}{\lambda}+\varepsilon+c(\varepsilon) T^{1 / r}+\frac{T^{\alpha}}{\lambda},
$$

where $0<\alpha<1-3 / r$ and $\varepsilon, c(\varepsilon)$ will be defined by (4.10).

Lemma 4.1. Let $f \in L_{r}\left(\Omega_{T}\right)$ and $h \in W^{1,0}\left(\Omega_{T}\right)$. Then

$$
L R(f, h)=(f, h)+T(f, h),
$$

where $T$ is a bounded operator with small norm if $\beta$ is small enough.

Proof. We have

$$
\begin{aligned}
L R(f, h) & =\sum_{k \in \mathcal{M} \cup \mathcal{N}}\left(L\left(x, t ; \partial_{x}\right)\left(\pi^{(k)} u^{(k)}, \pi^{(k)} \eta^{(k)}\right)-\pi^{(k)} L\left(x, t ; \partial_{x}\right)\left(u^{(k)}, \eta^{(k)}\right)\right) \\
& +\sum_{k \in \mathcal{M} \cup \mathcal{N}} \pi^{(k)}\left(L\left(x, t ; \partial_{x}\right)-L\left(\xi^{(k)}, 0 ; \partial_{x}\right)\right)\left(u^{(k)}, \eta^{(k)}\right) \\
& +\sum_{k \in \mathcal{M}} \pi^{(k)} L\left(\xi^{(k)}, 0 ; \partial_{x}\right)\left(u^{(k)}, \eta^{(k)}\right) \\
& +\sum_{k \in \mathcal{N}} \pi^{(k)} Z_{k}\left(L\left(\widehat{\xi}^{(k)}, 0 ; \partial_{z}-\nabla \bar{F} \partial_{z}\right)-L\left(\widehat{\xi}^{(k)}, 0 ; \partial_{z}\right)\right) Z_{k}^{-1}\left(u^{(k)}, \eta^{(k)}\right) \\
& +\sum_{k \in \mathcal{N}} \pi^{(k)} Z_{k} L\left(\widehat{\xi}^{(k)}, 0 ; \partial_{z}\right) Z_{k}^{-1}\left(u^{(k)}, \eta^{(k)}\right)=(f, h)+T(f, h) .
\end{aligned}
$$


To estimate the operator $T$ we apply Propositions 2.3 and 2.4:

$$
\begin{aligned}
& \quad\left\|\sum_{k} L_{1}\left(x, t ; \partial_{x}\right)\left(\pi^{(k)}, \eta^{(k)}\right)-\pi^{(k)} L_{1}\left(x, t ; \partial_{x}\right)\left(u^{(k)}, \eta^{(k)}\right)\right\|_{L_{r}} \\
& \leq c\left\|\sum_{k}|\nabla \pi| \cdot\left|u^{(k)}\right|\right\|_{L_{r}}+c\left\|\sum_{k}|\nabla \pi| \cdot|\nabla u|\right\|_{L_{r}}+c\left\|\sum_{k}|\nabla \pi| \cdot|\eta|\right\|_{L_{r}} \\
& \leq c \frac{T^{1 / r}+T^{1 / 2}}{\lambda}\left\|\sum_{k}|u|\right\|_{W_{r}^{2,1}}+c \frac{T^{\alpha}}{\lambda}\left\|\sum_{k}|\eta|\right\|_{V_{r}} \leq c \beta\left(\|f\|_{L_{r}}+\|h\|_{W_{r}^{1,0}}\right)
\end{aligned}
$$

and

$$
\begin{aligned}
& \left\|\sum_{k} L_{2}\left(x, t ; \partial_{x}\right)(\pi u, \pi \eta)-\pi L_{2}\left(x, t ; \partial_{x}\right)(u, \eta)\right\|_{W_{r}^{1,0}} \\
& \leq c\left\|\sum_{k}|\nabla \pi| \cdot|u|\right\|_{W_{r}^{1,0}} \leq c \frac{T^{1 / r}+T^{1 / 2}}{\lambda}\left\|\sum_{k}|u|\right\|_{W_{r}^{2,1}} \\
& \leq c \beta\left(\|f\|_{L_{r}}+\|g\|_{W_{r}^{1,0}}\right)
\end{aligned}
$$

and

$$
\begin{aligned}
& \left.\| \sum_{k} \pi\left(L_{1}\left(x, t ; \partial_{x}\right)-L_{1}\left(\xi, 0 ; \partial_{x}\right)\right)(u, \eta)\right) \|_{L_{r}} \\
& \leq c \lambda^{\alpha}\left(\left\|\sum_{k}|u|\right\|_{W_{r}^{2,1}}+\left\|\sum_{k}|\eta|\right\|_{W_{r}^{1,0}}\right) \leq c \beta\left(\|f\|_{L_{r}}+\|g\|_{W_{r}^{1,0}}\right)
\end{aligned}
$$

and

$$
\begin{aligned}
\| \sum_{k} \pi\left(L_{2}\left(x, t ; \partial_{x}\right)\right. & \left.-L_{2}\left(\xi, 0 ; \partial_{x}\right)\right)(u, \eta) \|_{W_{r}^{1,2}} \\
& \leq c \lambda^{\alpha}\left\|\sum_{k}|\nabla u|\right\|_{W_{r}^{1,0}} \leq c \beta\left(\|f\|_{L_{r}}+\|g\|_{W_{r}^{1,0}}\right)
\end{aligned}
$$

and

$$
\begin{aligned}
& \quad\left\|\sum_{k \in \mathcal{N}} \pi Z_{k}\left[\left(L_{1}\left(\widehat{\xi}, 0 ; \partial_{z}-\nabla \bar{F} \partial_{z}\right)-L_{1}\left(\widehat{\xi}, 0 ; \partial_{z}\right)\right) Z_{k}^{-1}(u, \eta)\right]\right\|_{L_{r}} \\
& \leq c\left\|\sum_{k}\left|\nabla^{2} \bar{F}\right| \cdot|\nabla u|\right\|_{L_{r}}+c\left\|\sum_{k}|\nabla \bar{F}| \cdot\left|\nabla^{2} u\right|\right\|_{L_{r}}+c\left\|\sum_{k}|\nabla \bar{F}| \cdot|\eta|\right\|_{L_{r}} \\
& \leq c \beta\left(\|f\|_{L_{r}}+\|g\|_{W_{r}^{1,0}}\right)+c\left\|\sum_{k}\left|\nabla^{2} \bar{F}\right| \cdot|\nabla u|\right\|_{L_{r}} .
\end{aligned}
$$

To estimate the last term we note that

$$
\|\nabla u\|_{L_{r}\left(0, T ; L_{\infty}(\Omega)\right)} \leq \varepsilon\|u\|_{W_{r}^{2,1}\left(\Omega_{T}\right)}+c(\varepsilon)\|u\|_{L_{r}\left(\Omega_{T}\right)},
$$


hence

$$
\begin{aligned}
\left\|\sum_{k}\left|\nabla^{2} \bar{F}\right| \cdot|\nabla u|\right\|_{L_{r}} & \leq c\left(\varepsilon+c(\varepsilon) T^{1 / r}\right)\left(\|f\|_{L_{r}}+\|g\|_{W_{r}^{1,0}}\right) \\
& \leq c \beta\left(\|f\|_{L_{r}}+\|g\|_{W_{r}^{1,0}}\right) .
\end{aligned}
$$

The same holds for the operator $L_{2}$. Summing up (4.5)-(4.10) we get

$$
\|T\| \leq c \beta \text {. }
$$

LEMMA 4.2. Let $u \in W_{r}^{2,1}\left(\Omega_{T}\right)$ and $\eta \in V(\Omega)$. Then

$$
R L(u, \eta)=(u, \eta)+W(u, \eta),
$$

where $W$ is a bounded operator with small norm if $\beta$ is small enough.

Proof. We have

$$
\begin{aligned}
R L(u, \eta)= & \sum_{k \in \mathcal{N}} \pi Z_{k} R^{(k)}\left[Z^{-1} \zeta^{(k)} L\left(x, t ; \partial_{x}\right)(u, \eta)\right] \\
& +\sum_{k \in \mathcal{M}} \pi R^{(k)}\left[\zeta^{(k)} L\left(x, t ; \partial_{x}\right)(u, \eta)\right] \\
= & \sum_{k \in \mathcal{N}} \pi Z R\left[Z^{-1}\left(\zeta L\left(x, t ; \partial_{x}\right)(u, \eta)-L\left(x, t ; \partial_{x}\right)(\zeta u, \zeta \eta)\right)\right] \\
& +\sum_{k \in \mathcal{N}} \pi Z R\left[Z^{-1}\left(L\left(x, t ; \partial_{x}\right)-L\left(\xi, 0 ; \partial_{x}\right)\right)(\zeta u, \zeta \eta)\right] \\
& +\sum_{k \in \mathcal{N}} \pi Z R\left[\left(L\left(\widehat{\xi}, 0 ; \partial_{z}-\nabla \bar{F} \partial_{z}\right)-L\left(\widehat{\xi}, 0 ; \partial_{z}\right)\right) Z^{-1}(\zeta u, \zeta \eta)\right] \\
& +\sum_{k \in \mathcal{M}} \pi R\left(\zeta L\left(x, t ; \partial_{x}\right)(u, \eta)-L\left(x, t ; \partial_{x}\right)(\zeta u, \zeta \eta)\right) \\
& +\sum_{k \in \mathcal{M}} \pi R\left(L\left(x, t ; \partial_{x}\right)-L\left(\xi, 0 ; \partial_{x}\right)\right)(\zeta u, \zeta \eta) \\
& +\sum_{k \in \mathcal{N}} \pi Z R L\left(\widehat{\xi}, 0 ; \partial_{z}\right) Z^{-1}(\zeta u, \zeta \eta)+\sum_{k \in \mathcal{M}} \pi R L\left(\xi, 0 ; \partial_{x}\right)(\zeta u, \zeta \eta) \\
= & (u, \eta)+W(u, \eta) .
\end{aligned}
$$

To estimate $W$ it is enough to repeat the considerations for the operator $T$ from Lemma 4.1 to get

$$
\|W\| \leq c \beta
$$

From Lemmas 4.1 and 4.2 we have

LEMMA 4.3. Let $f \in L_{r}\left(\Omega_{T}\right), h \in W_{r}^{1,0}\left(\Omega_{T}\right), g \in W_{r}^{2-1 / r, 1-1 /(2 r)}\left(S_{T}\right)$. Then there exists a unique solution of problem (4.2) such that $u \in W_{r}^{2,1}\left(\Omega_{T}\right)$, $\eta \in V\left(\Omega_{T}\right)$ and the following estimate holds: 


$$
\begin{aligned}
& \|u\|_{W_{r}^{2,1}\left(\Omega_{T}\right)}+\|\eta\|_{W_{r}^{1,0}\left(\Omega_{T}\right)}+\left\|\eta_{t}\right\|_{W_{r}^{1,0}\left(\Omega_{T}\right)} \\
& \quad \leq c\left(\|f\|_{L_{r}\left(\Omega_{T}\right)}+\|h\|_{W_{r}^{1,0}\left(\Omega_{T}\right)}+\|g\|_{W_{r}^{2-1 / r, 1-1 /(2 r)}\left(S_{T}\right)}\right)
\end{aligned}
$$

if $T$ is small enough.

Proof. First we take small $\lambda$ and $T \leq T_{*}(\lambda)$ (see (4.3)) such that $\beta$ is so small that (see (4.11) and (4.13))

$$
\|T\|<1 / 2, \quad\|W\|<1 / 2 .
$$

Then from (4.4) and (4.12), that is,

$$
L R \bar{d}=\bar{d}+T \bar{d}, \quad R L(u, \eta)=(u, \eta)+W(u, \eta),
$$

we get

$$
L R(1+T)^{-1} \bar{d}=\bar{d}, \quad(1+W)^{-1} R L(u, \eta)=(u, \eta) .
$$

By (4.15) the operators $(1+T)^{-1}$ and $(1+W)^{-1}$ are well defined, thus the inverse to $L$ is given by

$$
(1+W)^{-1} R=R(1+T)^{-1}=L^{-1},
$$

which gives the solution

$$
(u, \eta)=L^{-1} \bar{d}
$$

and the estimate (4.14) comes from Lemma 3.5 and the boundedness of $R$.

5. Proof of Theorem 1. To show Theorem 1 we have to reduce problem (1.1) to (4.1). We define extensions $\bar{u}_{0} \in W_{r}^{2,1}\left(\Omega_{T}\right)$ and $\bar{\eta}_{0} \in V\left(\Omega_{T}\right)$ of the initial data where

$$
\begin{array}{rlrl}
\left.\bar{u}_{0}\right|_{t=0} & =u_{0}, & \left\|\bar{u}_{0}\right\|_{W_{r}^{2,1}\left(\Omega_{T}\right)} & \leq c\left\|u_{0}\right\|_{W_{r}^{2-2 / r}(\Omega)}, \\
\left.\bar{\eta}_{0}\right|_{t=0} & =\eta_{0}, \quad\left\|\bar{\eta}_{0}\right\|_{V(\Omega)} \leq c\left\|\eta_{0}\right\|_{W_{r}^{1}(\Omega) .}
\end{array}
$$

If $u$ and $\eta$ are written in the form

$$
u=u^{\prime}+\bar{u}_{0}, \quad \eta=\eta^{\prime}+\bar{\eta}_{0},
$$

we obtain

$$
\begin{aligned}
& C u_{t}^{\prime}-\mu \Delta u^{\prime}-\nu \nabla \operatorname{div} u^{\prime}+A \eta^{\prime}=F^{\prime}, \\
& \eta_{t}^{\prime}+B \operatorname{div} u^{\prime}=H^{\prime}, \\
& \left.u^{\prime}\right|_{S_{T}}=G^{\prime}, \\
& \left.u^{\prime}\right|_{t=0}=0,\left.\quad \eta^{\prime}\right|_{t=0}=0,
\end{aligned}
$$

where by (5.1) we have 


$$
\begin{aligned}
& F^{\prime}=F-\left(C \bar{u}_{0, t}-\mu \Delta \bar{u}_{0}-\nu \nabla \operatorname{div} \bar{u}_{0}+A \bar{\eta}_{0}\right) \\
& H^{\prime}=H-B \operatorname{div} \bar{u}_{0}, \\
& G^{\prime}=G-\bar{u}_{0}, \\
& \left\|F^{\prime}\right\|_{L_{r}\left(\Omega_{T}\right)} \leq\|F\|_{L_{r}\left(\Omega_{T}\right)}+c\left\|u_{0}\right\|_{W_{r}^{2-2 / r}(\Omega)}+c\left\|\eta_{0}\right\|_{W_{r}^{1}(\Omega)}, \\
& \left\|H^{\prime}\right\|_{W_{r}^{1,0}\left(\Omega_{T}\right)} \leq\|H\|_{W_{r}^{1,0}\left(\Omega_{T}\right)}+c\left\|u_{0}\right\|_{W_{r}^{2-2 / r}(\Omega)}, \\
& \left\|G^{\prime}\right\|_{W_{r}^{2-1 / r, 1-1 /(2 r)}\left(S_{T}\right)} \leq\|G\|_{W_{r}^{2-1 / r, 1-1 /(2 r)}\left(S_{T}\right)}+c\left\|u_{0}\right\|_{W_{r}^{2-2 / r}(\Omega)} .
\end{aligned}
$$

By Lemma 4.3 and (5.4) we have a solution of (5.3), hence by (5.2) and (5.1) we have a solution of (1.1) with the estimate (1.2) on the time interval $\left[0, T_{*}\right]$, where $T_{*}$ is the $T$ from Lemma 4.3 , and we see that it is fixed. To end the proof we prolong the solution onto the intervals $\left[T_{*}, 2 T_{*}\right],\left[2 T_{*}, 3 T_{*}\right]$, etc. The proof of Theorem 1 is finished.

\section{References}

[1] O. V. Besov, V. P. Il'in and S. M. Nikol'skiǔ, Integral Representations of Functions and Imbedding Theorems, Nauka, Moscow, 1975 (in Russian).

[2] J. Marcinkiewicz, Sur les multiplicateurs des séries de Fourier, Studia Math. 8 (1939), 78-91.

[3] S. G. Mikhlin, Multidimensional Singular Integrals and Integral Equations, Pergamon Press, 1965.

[4] P. B. Mucha and W. Zajaczkowski, On the existence for the Cauchy-Neumann problem for the Stokes system in the $L_{p}$-framework, Studia Math. 143 (2000), 75-101.

[5] H. Triebel, Interpolation Theory, Function Spaces, Differential Operators, NorthHolland, Amsterdam, 1978.

Institute of Applied Mathematics and Mechanics Warsaw University

Banacha 2

02-097 Warszawa, Poland

E-mail: mucha@hydra.mimuw.edu.pl
Institute of Mathematics Polish Academy of Sciences Śniadeckich 8 00-950 Warszawa, Poland E-mail: wz@impan.gov.pl 